\title{
Go Games on Plasmodia of Physarum Polycephalum
}

\author{
Andrew Schumann \\ University of Information \\ Technology and Management in \\ Rzeszow, \\ ul. Sucharskiego 2, 35-225 \\ Rzeszow, Poland \\ Email: \\ andrew.schumann@gmail.com
}

\begin{abstract}
We simulate the motions of Physarum polycephalum plasmodium by the game of Go, the board game originated in ancient China more than 2,500 years ago. Then we concentrate just on Go games, where locations of black and white stones simulate syllogistic reasoning, in particular reasoning of Aristotelian syllogistic and reasoning of performative syllogistic. For the first kind of reasoning we need a special form of coalition games. For the second kind of reasoning we appeal to usual antagonistic games.
\end{abstract}

\section{INTRODUCTION}

$\mathrm{I}$ N the Physarum Chip Project: Growing Computers From Slime Mould [1] we are working on designing a biological computer, where logic circuits are represented by programmable behaviors of Physarum polycephalum plasmodium, the one-cell organism that behaves according to different chemical stimuli called attractants and repellents and propagates networks connecting all reachable food attractants [2], [12]. The behavior of plasmodia is very sensitive and intelligent [4], [5], [6], [7], [8], [15], [17], [18], [19]. This behavior can be represented as a bio-inspired game theory on plasmodia [14], i.e. an experimental game theory, where, on the one hand, all basic definitions are verified in the experiments with Physarum polycephalum and Badhamia utricularis and, on the other hand, all basic algorithms are implemented in the object-oriented language for simulations of plasmodia [13].

We show that the slime mold can be a model for concurrent games and context-based games defined in [11]. In context-based games, players can move concurrently as well as in concurrent games, but the set of actions is ever infinite. In our experiments, we follow the following interpretations of basic entities: (1) attractants as payoffs; (2) attractants occupied by the plasmodium as states of the game; (3) active zones of plasmodium as players; (4) logic gates for behaviors as moves (available actions) for the players; (5) propagation of the plasmodium as the transition table which associates, with a given set of states and a given move of the players, the set of states resulting from that move.
In this game theory we can demonstrate creativity of primitive biological substrates of plasmodia. The point is that plasmodia do not strictly follow spatial algorithms like Kolmogorov-Uspensky machines, but perform many additional actions. So, the plasmodium behavior can be formalized within strong extensions of spatial algorithms, e.g. within concurrent games or context-based games [11].

In this paper we show how we can represent the plasmodium behavior as a Go game. It is board game with two players (called Black and White) who alternately place black and white stones, accordingly, on the vacant intersections (called points) of a board with a 19x19 grid of lines. Black moves first. Stones are placed until they reach a point where stones of another color are located. There are the following two basic rules of the game: (1) each stone must have at least one open point (called liberty) directly next to it (up, down, left, or right), or must be part of a connected group that has at least one such open point; stones which lose their last liberty are removed from the board; (2) the stones must never repeat a previous position of stones. The aim of the game is in surrounding more empty points by player's stones. At the end of game, the number of empty points player's stones surround are counted, together with the number of stones the player captured. This number determines who the winner is. The Go game originated in ancient China more than 2,500 years ago and it is very popular till now.

We can consider the game of Go as a model of plasmodium motions. In this view the black stones are considered attractants occupied by the plasmodium and the white stones are regarded as repellents. By this interpretation, we have two players, also: Black (this player places attractants) and White (this player places repellents). The winner is determined by the number of empty points player's stones surround.

Notice that the number of possible Go games is too large, $10^{761}$. Therefore it is better to focus just on games, where locations of black and white stones simulate spatial reasoning. In this paper we propose two logics in the universe of possible Go games: (1) Aristotelian syllogistic [3]; (2) performative syllogistic [9], [10]. 


\section{Classical GaMe OF Go ON Plasmodia OF PHYSARUM POLYCEPHALUM}

The plasmodium of Physarum polycephalum moves to attractants to connect them and in the meanwhile it avoids places, where repellents are located. The radius, where chemical signals from attractants (repellents) can be detected by the plasmodium to attract (repel) the latter, determines the structure of natural Voronoi cells, where each Voronoi cell is a place, where a chemical signal holds (see Fig. 1).

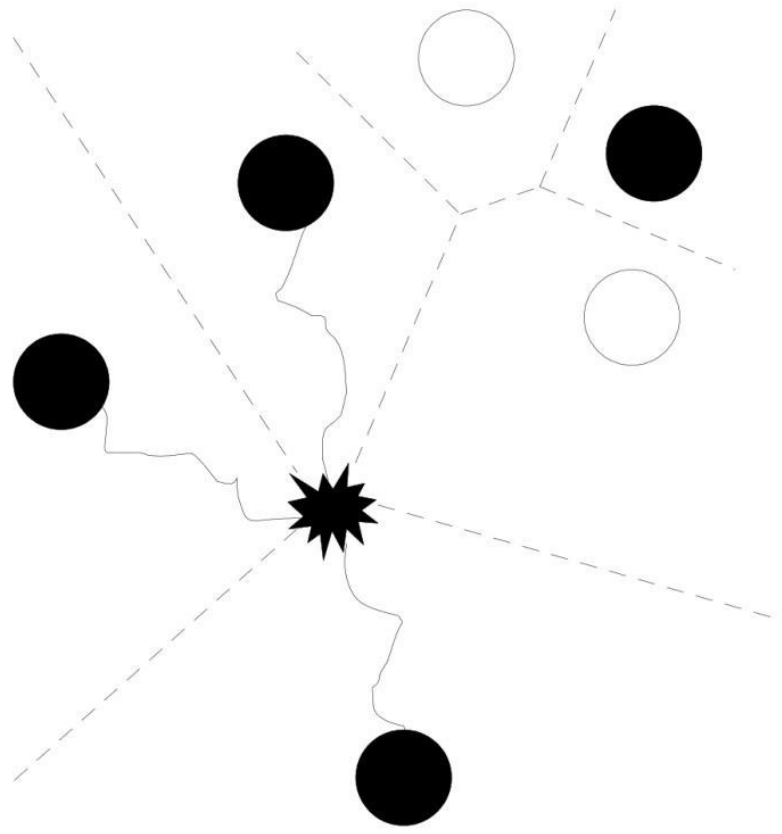

Fig. 1 The six Voronoi cells in accordance with the four attractants denoted by the black stones and with the two repellents denoted by the white stones. The plasmodium located in the center of the picture connects the three attractants by the three protoplasmic tubes. It cannot see the fourth attractant because of the two repellents

For the plasodium of Fig. 1 we have just the four neighbor cells. Notably that in Go games at each point we have only four neighbors everywhere. So, we can design the space for plasmodia in the way to have just four neighbors at each point. So, the cells of a Go game board are considered Voronoi cells with the same radius of intensity and power of attractants and repellents located in these cells. We associate black stones with attractants occupied by plasmodia and white stones with repellents. For the sake of convenience and more analogy with Voronoi cells, let us consider cells (not intersections of lines) as points for stone locations (see Fig. 2). Then we can use all rules of Go games to simulate plasmodium motions.

Thus, our Go game is represented in the universe of 18x18 Voronoi cells (see Fig. 2).

\section{ARISTOTELIAN Go GAME ON Plasmodia}

Let us assume that the game of Go is a coalitional game with two players who choose only strategies to place black and white stones so that their locations can be interpreted as a spatial Aristotelian syllogistic reasoning.

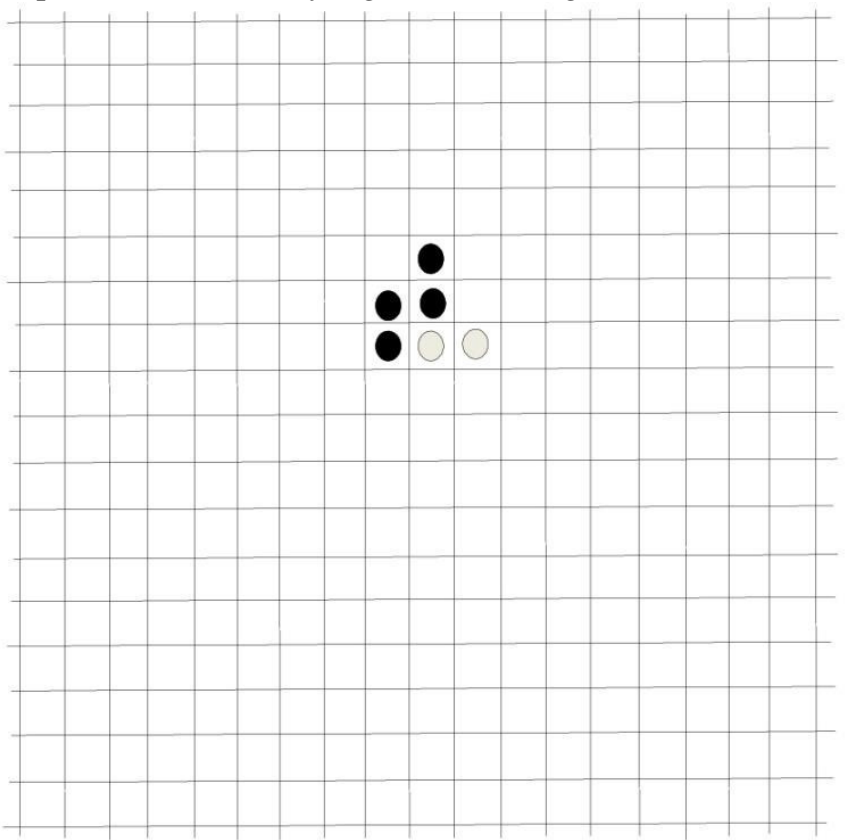

Fig. 2 The Go game board with two white stones designating repellents and four black stones designating attractants

Let us recall that the axiomatization of the Aristotelian syllogistic was laid first by Łukasiewicz [3]. In his axiomatization, the alphabet consists of the syllogistic letters $S, P, M, \ldots$, the syllogistic connectives $a, e, i, o$, and the propositional connectives $\neg, \vee, \wedge, \Rightarrow$. Atomic propositions are defined as follows: $\operatorname{SxP}$, where $x \in\{a, e, i$, $o\}$. All other propositions are defined in the following way: (i) each atomic proposition is a proposition, (ii) if $X, Y$ are propositions, then $\neg X, \neg Y, X$ å $Y$, where $a ̊\{\vee, \wedge, \Rightarrow\}$, are propositions, also. The axioms proposed by Łukasiewicz are as follows:

$$
\begin{gathered}
S a P:=(\exists A(A \mathrm{i} s S) \wedge \forall A(A \mathrm{i} s S \Rightarrow A \mathrm{i} s P)) ; \\
\operatorname{SiP}:=\exists A(A \mathrm{i} s S \wedge A \mathrm{i} s P) ; \\
\operatorname{SeP}:=\neg(\operatorname{SiP}) ; \\
S o P:=\neg(\operatorname{SaP}) ;
\end{gathered}
$$

$S a S$

SiS ;

$$
(M a P \wedge S a M) \Rightarrow S a P
$$

$(M a P \wedge M i S) \Rightarrow S i P$. 
In the Go implementation of Aristotelian syllogistic, the syllogistic letters $S, P, M, \ldots$ are interpreted as single cells of the board with the $18 \times 18$ Voronoi cells. The letter $S$ is understood as empty if and only if the white stone is located on an appropriate cell denoted by $S$. Let us recall that in our plasmodium interpretation of Go games white stones mean ever repellents so that their location in a Voronoi cell means that this cell cannot be occupied by plasmodia. This letter $S$ is treated as non-empty if and only if the black stone is located on an appropriate cell denoted by $S$. Recall that black stones mean ever attractants so that their location in a Voronoi cell means that this cell is occupied by plasmodia. If a cell does not contain any stone, this means that this cell is out of the game.

Hence, in the Physarum interpretation of this Go game, the non-empty syllogistic letters $S, P, M, \ldots$, i.e. the cells denoted by $S, P, M, \ldots$ containing black stones are considered attractants and the empty syllogistic letters $S, P$, $M, \ldots$, i.e. the cells denoted by $S, P, M, \ldots$ containing white stones are considered repellents. So, a data point $S$ is regarded as non-empty if and only if an appropriate attractant located in $S$ is occupied by plasmodium. This data point $S$ is regarded as empty if and only if an appropriate repellent located in $S$ repell plasmodium.

Thus, in the Aristotelian version of the Go game we have syllogistic strings of the form $S P$ with the following interpretation: ' $S$ is $P$ ', and with the following meaning: $S P$ is true if and only if $S$ and $P$ are neighbors and both $S$ and $P$ are not empty, otherwise $S P$ is false. We can extend this meaning as follows: $S P$ is true if and only if $S$ and $P$ are not empty and there is a line of non-empty cells between points $S$ and $P$, otherwise $S P$ is false. By the definition of true syllogistic strings, we can define atomic syllogistic propositions as follows:

In the formal syllogistic: there exists $A$ such that $A$ is $S$ and for any $A$, if $A$ is $S$, then $A$ is $P$. In the Go game model: there is a cell $A$ containing the black stone and for any $A$, if $A S$ is true, then $A P$ is true. In the Physarum model: there is a plasmodium in the cell $A$ and for any $A$, if $A S$ is true, then $A P$ is true.

In the formal syllogistic: there exists $A$ such that both $A S$ is true and $A P$ is true. In the Go game model: there exists a cell $A$ containing the black stone such that $A S$ is true and $A P$ is true. In the Physarum model: there exists a plasmodium in the cell $A$ such that $A S$ is true and $A P$ is true.

In the formal syllogistic: for all $A, A S$ is false or $A P$ is false. In the Go game model: for all cells $A$ containing the black stones, $A S$ is false or $A P$ is false. In the Physarum model: for all plasmodia $A, A S$ is false or $A P$ is false.

In the formal syllogistic: for any $A, A S$ is false or there exists $A$ such that $A S$ is true and $A P$ is false. In the Go game model: for all cells $A$ containing the black stones, $A S$ is false or there exists $A$ such that $A S$ is true and $A P$ is false. In the Physarum model: for any plasmodia $A, A S$ is false or there exists $A$ such that $A S$ is true and $A P$ is false.
Formally, this semantics is defined as follows. Let $M$ be a set of attractants. Take a subset $|X| \subseteq M$ of cells containing the black stones (i.e. of cells containing attractants and occupied by the plasmodium) as a meaning for each syllogistic variable $X$. Next, define an ordering relation $\subseteq$ on subsets $|S|,|P| \subseteq M$ as: $|S| \subseteq|P|$ iff all attractants from $|P|$ are reachable for the plasmodium located at the attractants from $|S|$, i.e. iff for all cells of $|S|$ with black stones there are lines of black stones connecting them to cells of $|P|$ also containing black stones. Hence, $|S| \cap|P| \neq \varnothing$ means that some attractants from $|P|$ are reachable for the plasmodium located at the attractants from $|S|$ and $|S| \cap|P|=\varnothing$ means that no attractants from $|P|$ are reachable for the plasmodium located at the attractants from $|S|$. In the Go game model $|S|$ $\cap|P| \neq \varnothing$ means that some cells from $|P|$ occupied by the black stones are connected by the lines of black stones with the cells from $|S|$ occupied by the black stones and $|S| \cap|P|=$ $\varnothing$ means that there are no lines of black stones from the cells of $|P|$ to the cells of $|S|$.

This gives rise to models $\mathrm{M}=\langle M,|\cdot|\rangle$ such that

$$
\begin{aligned}
& \mathrm{M} \mid=S a P \text { iff }|S| \subseteq|P| ; \\
& \mathrm{M} \mid=S i P \text { iff }|S| \cap|P| \neq \varnothing ; \\
& \mathrm{M} \mid=S e P \text { iff }|S| \cap|P|=\varnothing ; \\
& \mathrm{M} \mid=p \wedge q \text { iff } \mathrm{M} \mid=p \text { and } \mathrm{M} \mid=q ; \\
& \mathrm{M} \mid=p \vee q \text { iff } \mathrm{M} \mid=p \text { or } \mathrm{M} \mid=q ; \\
& \mathrm{M} \mid=\neg p \text { iff it is false that } \mathrm{M} \mid=p
\end{aligned}
$$

Proposition 1: The Aristotelian syllogistic is sound and complete relatively to $\mathrm{M}$ if we understand $\subseteq$ as an inclusion relation (it is a well-known result [16]).

However, relatively to all possible Go games (plasmodium behaviors) the Aristotelian syllogistic is not complete. Indeed, the relation $\subseteq$ can have the following verification on the Aristotelian Go game model on plasmodia according to our definitions: $|S| \subseteq|P|$ and $|S| \subseteq$ $\left|P^{\prime}\right|$, where $|P| \cap\left|P^{\prime}\right|=\varnothing$, i.e. all attractants from $|P|$ are reachable for the plasmodium located at the attractants from $|S|$ and all attractants from $\left|P^{\prime}\right|$ are reachable for the plasmodium located at the attractants from $|S|$, but between $|P|$ and $\left|P^{\prime}\right|$ there are no paths. In this case $\subseteq$ is not an inclusion relation and proposition 1 does not hold. Hence, we need repellents to make $\subseteq$ the inclusion relations in all cases. Therefore, to obtain the Aristotelian Go game model on plasmodia we shall deal with the coalitional game, where two players will cooperate to build spatial reasoning satisfying the rules of Aristotelian syllogistic. The first player places black stones to designate places of growing plasmodia. The second player places white stones to 
designate places of repelling plasmodia. So, both players follow coalitional strategies to simulate Aristotelian syllogistic reasoning.

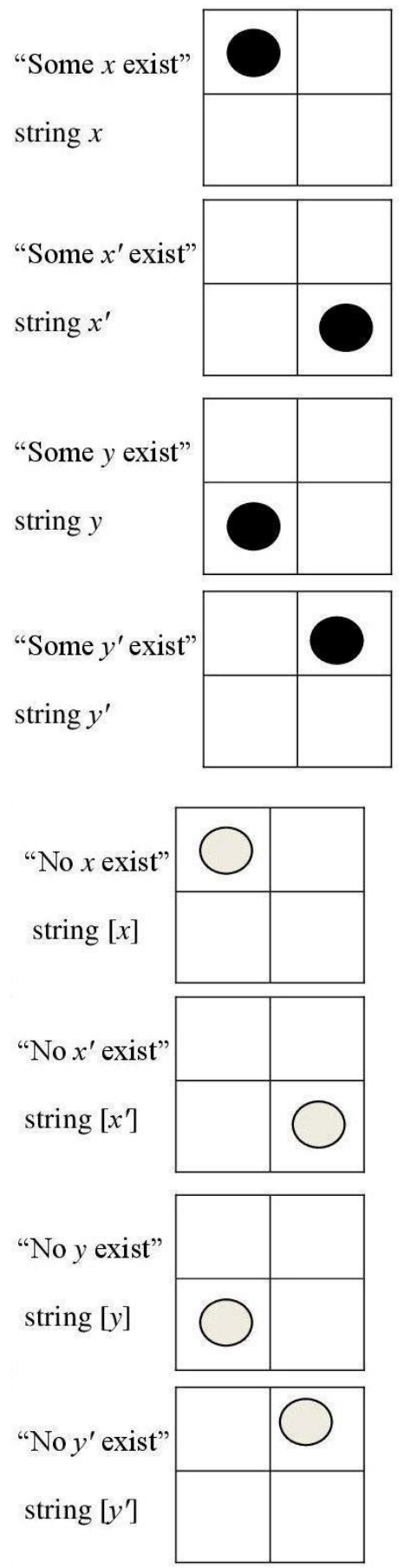

Fig. 3 The Aristotelian Go game diagrams for the basic existence strings

\section{A. Coalition Go Game for Verifying Aristotelian Reasoning}

In the Aristotelian Go game model for verifying all the basic syllogistic propositions, we will use the following four cells: $x, y, x^{\prime}, y^{\prime}$ of the game board with the 19x19 grid of lines, where $x^{\prime}$ means all cells which differ from $x$, but they are neighbors for $y$, and $y^{\prime}$ means all cells which differ from $y$ and are neighbors for $x$. These cells express appropriate meanings of syllogistic letters. The corresponding universe of discourse will be denoted by means of the following diagram:

\begin{tabular}{|l|l|}
\hline$x$ & $y^{\prime}$ \\
\hline$y$ & $x^{\prime}$ \\
\hline
\end{tabular}

Assume that a black stone denotes an attractant and if it is placed within a cell $x$, this means that "this Voronoi cell contains an attractant $N_{x}$ activated and occupied by the plasmodium". It is a verification of the syllogistic letter $S_{x}$ at cell $x$ of the board. A white stone denotes a repellent and if it is placed within a cell $x$, this means that "this Voronoi cell contains a repellent $R_{x}$ activated and there is no plasmodium in it". It is a verification of a new syllogistic letter $\left[S_{x}\right]$. For the sake of convenience, we will denote $S_{x}$ by $x$ and $\left[S_{x}\right]$ by $[x]$. Using these stones, we can verify all the basic existence syllogistic propositions (see Fig. 3).

Aristotelian Go game strings of the form $x y, y x$ are interpreted as particular affirmative propositions "Some $x$ are $y$ " and "Some $y$ are $x$ " respectively, strings of the form $[x y],[y x], x[y], y[x]$ are interpreted as universal negative propositions "No $x$ are $y$ " and "No $y$ are $x$ ". A universal affirmative proposition "All $x$ are $y$ " are presented by a complex string $x y \& x\left[y^{\prime}\right]$. The sign \& means that we have strings $x y$ and $x\left[y^{\prime}\right]$ simultaneously and they are considered the one complex string. All these strings are verified on the basis of the diagrams of Fig. 4. So, we use only black stones for building particular propositions, only white stones for building universal negative propositions, and we combine black and white stones for building universal affirmative propositions. Consequently, we need a cooperation of two players to implement a spatial version of Aristotelian syllogistic within coalition Go games.

For verifying syllogisms we will use the following diagrams symbolizing some neighbor cells:

\begin{tabular}{|l|l|l|l|}
\hline & $m$ & $m^{\prime}$ & \\
\hline$m^{\prime}$ & $x$ & $y^{\prime}$ & $m$ \\
\hline$m$ & $y$ & $x^{\prime}$ & $m^{\prime}$ \\
\hline & $m^{\prime}$ & $m$ & \\
\hline
\end{tabular}

The motion of plasmodium starts from one of the central cells $\left(x, y, x^{\prime}, y^{\prime}\right)$ and goes towards one of the four directions (northwest, southwest, northeast, southeast). The syllogism shows a connection between two not-neighbor cells on the basis of its joint neighbor and says if there was either multiplication or fusion of plasmodia (i.e. either splitting or 
fusion of the lines of black stones). As a syllogistic conclusion, we obtain another diagram:

$$
\begin{array}{|c|c|}
\hline x & m^{\prime} \\
\hline m & x^{\prime} \\
\hline
\end{array}
$$

Different syllogistic conclusions derived show directions of plasmodium's propagation. Some examples are provided in Fig. $5-7$.
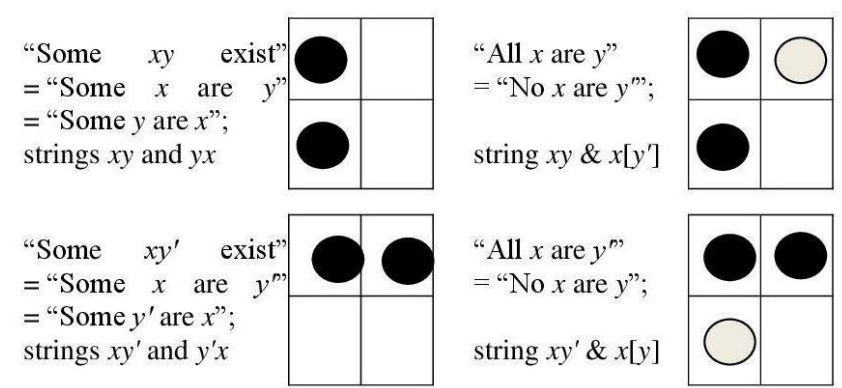

"All $x$ are $y$ "

= "No $x$ are $y$ ";

string $x y^{\prime} \& x[y]$
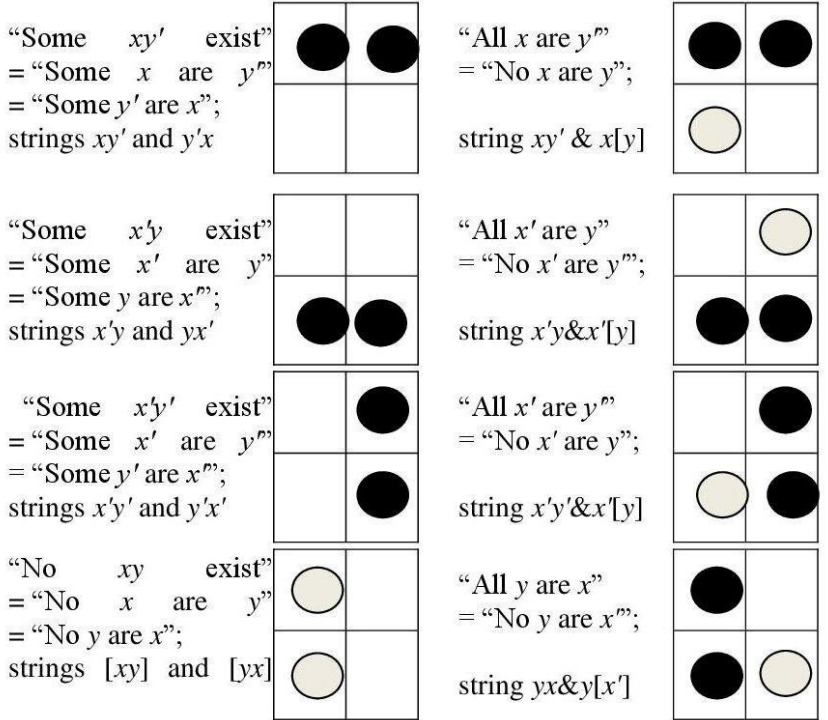

"All $x^{\prime}$ are $y$ "

$=$ "No $x^{\prime}$ are $y^{\prime \prime}$;

string $x^{\prime} y \& x^{\prime}[y]$

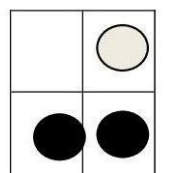

"All $x^{\prime}$ are $y$ "

$=$ "No $x^{\prime}$ are $y^{\prime \prime}$;

string $x^{\prime} y^{\prime} \& x^{\prime}[y]$

"All $y$ are $x$ "

= "No $y$ are $x$ ");

string $y x \& y\left[x^{\prime}\right]$
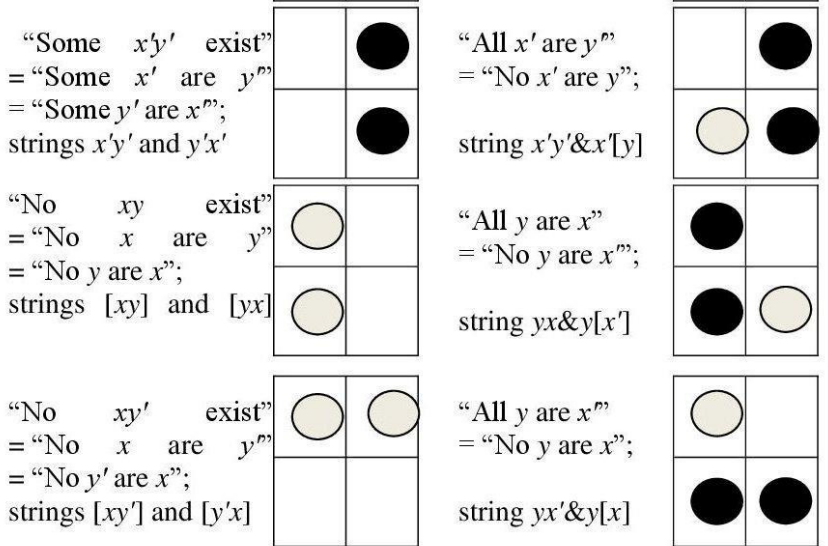

"All $y$ are $x$ ",

$=$ "No $y$ are $x "$ ";

string $y x^{\prime} \& y[x]$

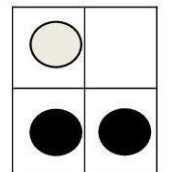

\begin{tabular}{lll|l|l|} 
"No $\quad x^{\prime} y \quad$ exist" & & \\
$=$ "No $x^{\prime}$ are $y "$ & & \\
= "No $y$ are $x " ;$ & & & \\
strings $\left[x^{\prime} y\right]$ and $[y x]$ & & & \\
\hline
\end{tabular}

$\begin{array}{llll}\text { "No } & x^{\prime} y^{\prime} & \text { exist" } \\ =\text { "No } & x^{\prime} & \text { are } & y^{m "}\end{array}$

$=$ "No $y^{\prime}$ are $x^{\prime \prime}$;

strings $\left[x^{\prime} y^{\prime}\right]$ and $\left[y^{\prime} x^{\prime}\right]$

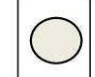

"Some $x$ are $y$ ",

"Some $x$ are $y$ ";

strings $x y, y x, x y^{\prime}, y^{\prime} x$

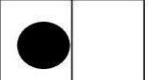

"Some $x^{\prime}$ are $y$ "

"Some $x^{\prime}$ are $y$ ";

strings $x^{\prime} y^{\prime}, y^{\prime} x^{\prime}, x^{\prime} y$ $y x^{\prime}$

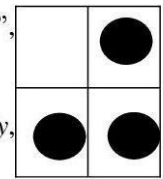

"All $y^{\prime}$ are $x$ "

$=$ "No $y^{\prime}$ are $x^{\prime "}$,

string $y^{\prime} x \& y^{\prime}\left[x^{\prime}\right]$

"All $y^{\prime}$ are $x^{\prime \prime}$

= "No $y^{\prime}$ are $x "$;

string $y^{\prime} x^{\prime} \& y^{\prime}[x]$
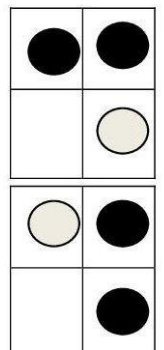

"Some $y$ are $x$ "

"Some $y$ are $x^{m "}$;

strings $x y, y x, x^{\prime} y$ $y x^{\prime}$

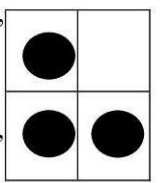

"Some $y^{\prime}$ are $x^{\prime}$

"Some $y^{\prime}$ are $x^{\prime \prime}$;

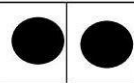

strings $x y^{\prime}, \quad y^{\prime} x$ $x^{\prime} y^{\prime}, y^{\prime} x^{\prime}$
1.

No $y$ are $x^{\prime}$;

All $y^{\prime}$ are $m$.

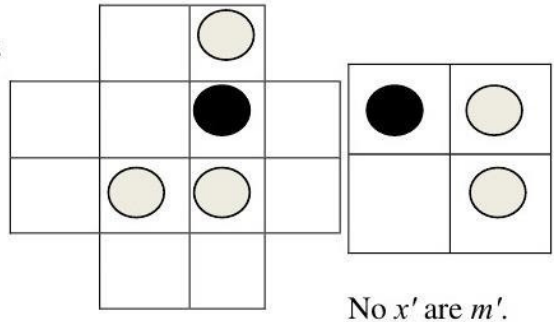

2. All $y^{\prime}$ are $x$; All $y^{\prime}$ are $m^{\prime}$.

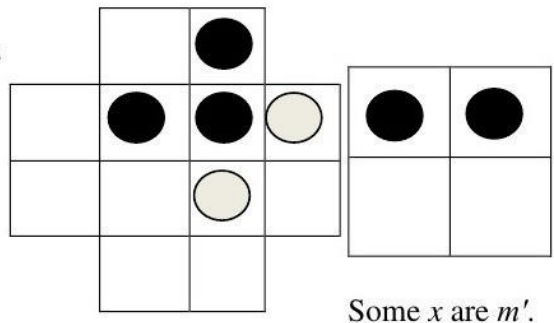

3. No $x^{\prime}$ are $y^{\prime}$;

Some $y$ are $m^{\prime}$.

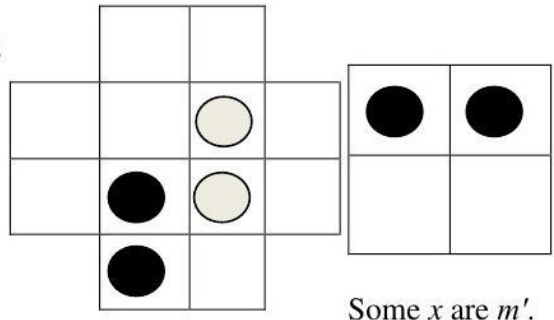

5. Some $y$ are $x^{\prime}$;

No $m$ are $y$.

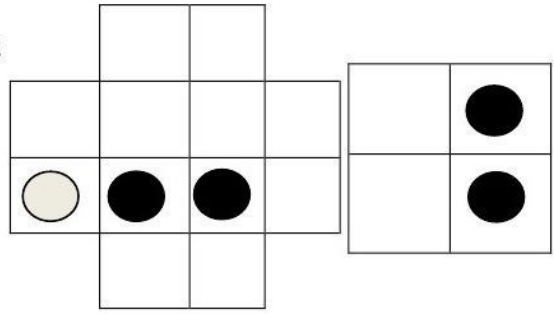

Some $x^{\prime}$ are $m^{\prime}$.

6. No $x^{\prime}$ are $m$;

No $m$ are $y$.

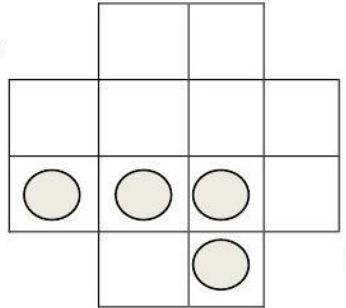

There is no conclus

7. No $y$ are $x^{\prime}$

Some $m^{\prime}$ are $y$.

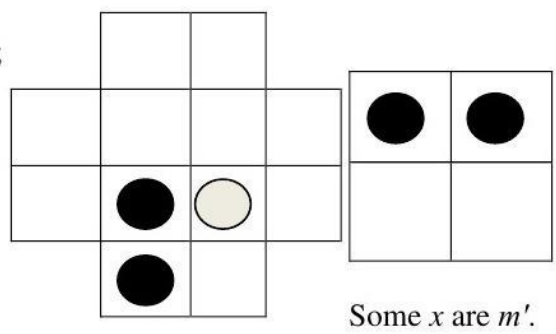

Fig. 5 The Aristotelian Go game diagrams for syllogisms (part 1)

Fig. 4 The Aristotelian Go game diagrams for syllogistic propositions 
8. All $y^{\prime}$ are $x^{\prime}$; No $y^{\prime}$ are $m$.

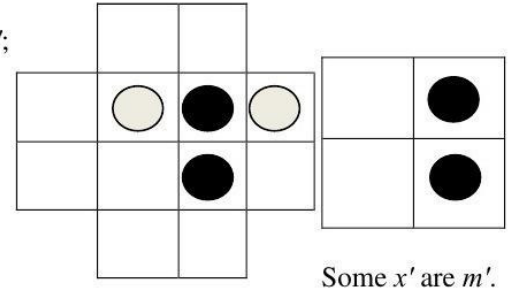

9. Some $x^{\prime}$ are $m^{\prime}$; No $m$ are $y^{\prime}$.

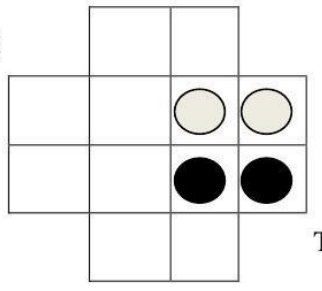

There is no conclusion.

10. All $y$ are $x$; All $y$ are $m$.

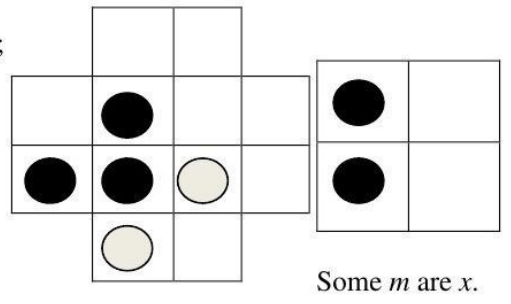

11. No $x^{\prime}$ are $m$; No $m^{\prime}$ are $y$.

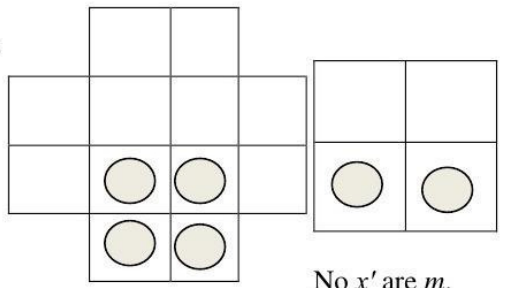

12. All $y^{\prime}$ are $x$; Some $m$ are $y^{\prime}$.

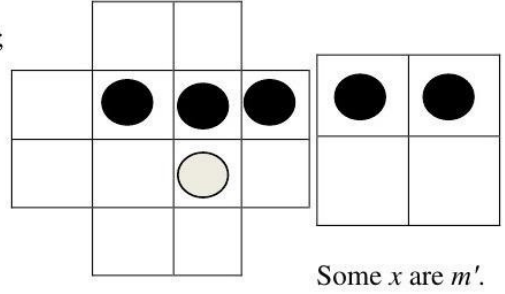

13. All $y$ are $m$; All $x$ are $y$.

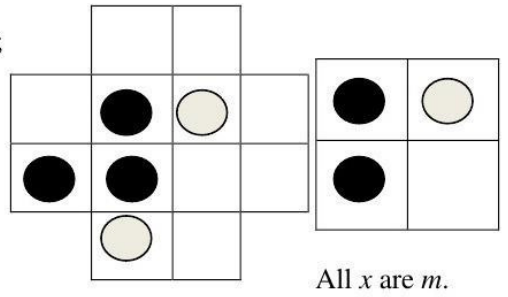

Fig. 6 The Aristotelian Go game diagrams for syllogisms (part 2)
14. Some $y$ are $x$; No $m^{\prime}$ are $y$.
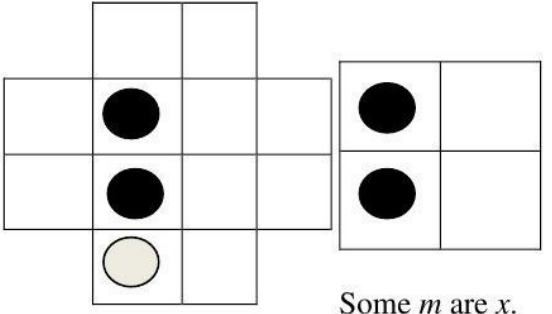

Some $m$ are $x$.

15. No $x$ are $y$; Some $m$ are $y$.
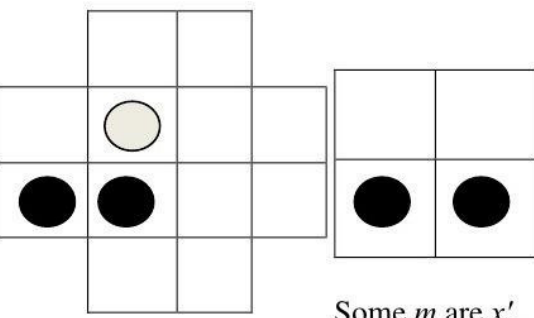

Some $m$ are $x^{\prime}$.

16. Some $y$ are $x$; All $y$ are $m^{\prime}$.

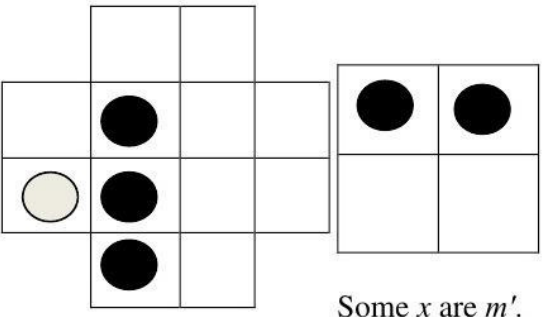

17. All $y$ are $x$; All $y^{\prime}$ are $m^{\prime}$.

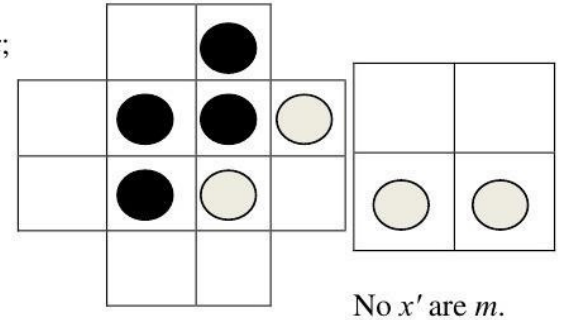

18. Some $x$ are $y$; All $y$ are $m$.

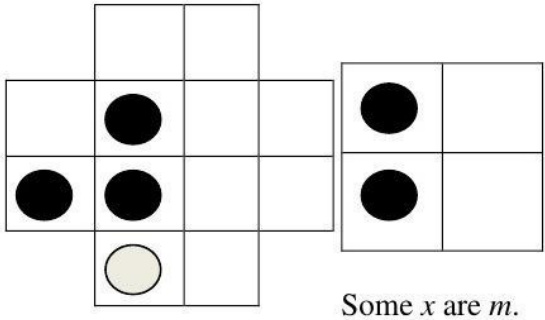

Fig. 7 The Aristotelian Go game diagrams for syllogisms (part 3)

Continuing in the same way, we can construct a syllogistic system, where conclusions are derived from three premises. The Aristotelian Go game (i.e. the suitable motion of plasmodium) starts from one of the central cells $\left(x, y, x^{\prime}\right.$, $y^{\prime}$ ) and goes towards one of the four directions (northwest, southwest, northeast, southeast), then towards one of the eight directions (north-northwest, west-northwest, southsouthwest, west-southwest, north-northeast, east-northeast, south-southeast, east-southeast), etc.

Hence, a Go game or spatial expansion of plasmodium is interpreted as a set of syllogistic propositions. The universal affirmative proposition $x y \quad \& \quad x[y]$ means that the 
plasmodium at the place $x$ goes only to $y$ and all other directions are excluded. The universal negative proposition $x[y]$ or $[x y]$ means that the plasmodium at the place $x$ cannot go to $y$ and we know nothing about other directions. The particular affirmative proposition $x y$ means that the plasmodium at the place $x$ goes to $y$ and we know nothing about other directions. Syllogistic conclusions allow us to mentally reduce the number of syllogistic propositions showing plasmodium's propagation.

For the implementation of Aristotelian syllogistic we appeal to repellents to delete some possibilities in the plasmodium propagation. So, model $M$ defined above should be understood as follows:

$$
\mathrm{M} \mid=A l l \quad x \text { are } y \text { iff } x y \quad \& \quad x\left[y^{\prime}\right] \text {, i.e. the }
$$

plasmodium is located at $x$ and can move only to $y$ and cannot move towards all other directions (the black stone is placed at $x$ and we can build the line of black stones only to $y)$;

$\mathrm{M} \mid=$ Some $x$ are $y$ iff $x y$, i.e. the plasmodium is located at $x$ and can move to $y$ (the black stone is placed at $x$ and we can build the line of black stones to $y$ );

$\mathrm{M} \mid=$ No $x$ are $y$ iff $x[y]$ or $[x y]$, i.e. the plasmodium cannot move to $y$ in any case (there is no line of black stones to $y$ ).

It is evident in this formulation that the Aristotelian syllogistic is so unnatural for plasmodia. Without repellents (the coalition game of two players), this syllogistic system cannot be verified in the medium of plasmodium propagations (Go game). In other words, we can prove the next proposition:

Proposition 2: The Aristotelian syllogistic is not sound and complete on the plasmodium without repellents. In other words, the Aristotelian syllogistic is not sound and complete in the Go game without a coalition of two players.

In other words, the Aristotelian syllogistic reasoning can be implemented as a Go game if and only if two players agree to play cooperatively to place black and white stones in accordance with spatial implementation of syllogisms.

\section{B. Examples of Aristotelian Go Game}

Let us consider a game of Go at time step 10, i.e. when White and Black players have placed the 10 white stones and the 10 black stones respectively. Let this game be pictured in Figure 8. Each Voronoi cell is denoted from $S_{1,1}$ to $S_{18,18}$. So, in Figure 8 syllogistic letters $S_{6,4}, S_{7,5}, S_{7,6}, S_{8,7}$, $S_{8,8}, S_{7,9}, S_{8,10}, S_{6,11}, S_{4,9}, S_{4,10}$ are understood as non-empty and syllogistic letters $S_{4,6}, S_{5,6}, S_{6,8}, S_{6,9}, S_{6,10}, S_{4,11}, S_{7,10}, S_{7,12}$, $S_{11,8}, S_{12,8}$ as empty. As a result, we can build some true syllogistic propositions in this universe like that: 'Some $S_{7,5}$ are $S_{7,6}$ ', 'Some $S_{8,7}$ are $S_{8,8}$ ', 'Some $S_{4,9}$ are $S_{4,10}$ ', 'No $S_{4,6}$ are $S_{5,6}$ ', 'No $S_{6,8}$ are $S_{6,9}$ ', 'No $S_{6,9}$ are $S_{6,10}$ ', 'No $S_{6,10}$ are $S_{7,10}$ ', 'No $S_{11,8}$ are $S_{12,8}$, etc.

Let us notice that in the universe of Fig. 8 we do not have universal affirmative propositions. But we can draw some syllogistic conclusions such as 'If $S_{4,10}\left[S_{4,11}\right]$ and $S_{4,9} S_{4,10}$, then $S_{4,9}\left[S_{4,11}\right]$ ' (i.e. 'If no $S_{4,10}$ are $S_{4,11}$ and some $S_{4,9}$ are $S_{4,10}$, then no $S_{4,9}$ are $S_{4,11}$ ').

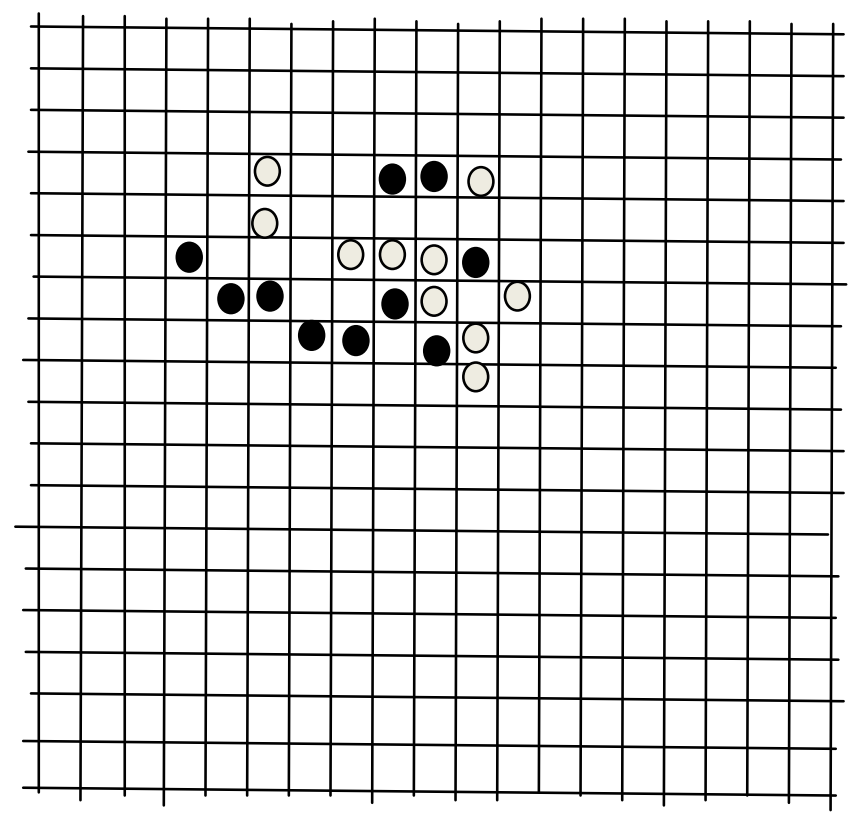

Fig. 8 The Aristotelian Go game 1 at time step 10

On the contrary, in the universe pictured in the Go game of Fig. 9 we have universal affirmative propositions such as 'All $S_{8,10}$ are $S_{8,11}$ ' and 'All $S_{4,9}$ are $S_{3,9}$ '. Some possible conclusions: 'If no $S_{8,11}$ are $S_{8,12}$ and all $S_{8,10}$ are $S_{8,11}$, then no $S_{8,10}$ are $S_{8,12}$ ' and 'If no $S_{3,9}$ are $S_{3,10}$ and all $S_{4,9}$ are $S_{3,9}$, then no $S_{4,9}$ are $S_{3,10}$ '.

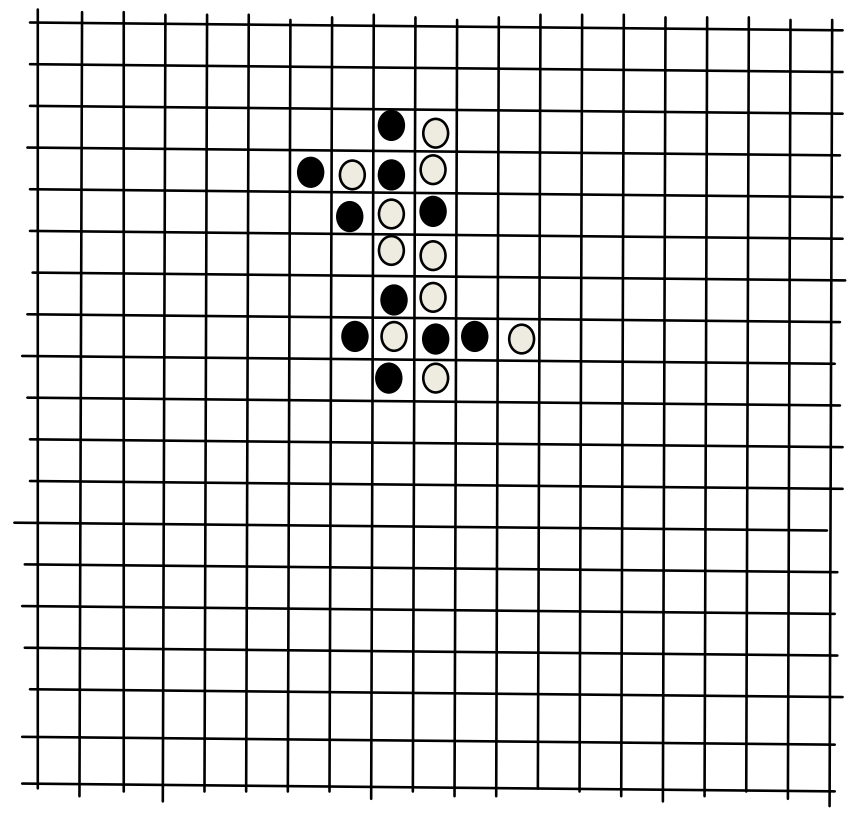

Fig. 9 The Aristotelian Go game 2 at time step 10 


\section{NON-ARISTOTELIAN SYLLOGISTIC Go GAME ON PLASMODIA}

While in Aristotelian syllogisms we are concentrating on one direction of many Physarum motions, and dealing with acyclic directed graphs with fusions of many protoplasmic tubes toward one data point, in most cases of Physarum behavior, not limited by repellents, we observe a spatial expansion of Physarum protoplasm in all directions with many cycles. Under these circumstances it is more natural to define all the basic syllogistic propositions $\mathrm{SaP}, \mathrm{SiP}, \mathrm{SeP}$, $S o P$ in a way they satisfies the inverse relationship when all converses are valid: $\mathrm{SaP} \Rightarrow \mathrm{PaS}, \mathrm{SiP} \Rightarrow P i S, \mathrm{SeP} \Rightarrow P e S$, $S o P \Rightarrow P o S$. In other words, we can draw more natural conclusions for protoplasmic tubes which are decentralized and have some cycles. The formal syllogistic system over propositions with such properties is constructed in [9], [10]. This system is called the performative syllogistic. The alphabet of this system contains as descriptive signs the syllogistic letters $S, P, M, \ldots$, as logical-semantic signs the syllogistic connectives $a, e, i, o$, and the propositional connectives $\neg, \vee, \wedge, \Rightarrow$. Atomic propositions are defined as follows: $S x P$, where $x \in\{a, e, i, o\}$. All other propositions are defined thus: (i) each atomic proposition is a proposition, (ii) if $X, Y$ are propositions, then $\neg X, \neg Y$, $X$ å $Y$, where $\mathrm{a} \in\{\vee, \wedge, \Rightarrow\}$, are propositions, too.

Let us consider Go games with the two different kinds of plasmodia: (i) plasmodia of Physarum polycephalum and (ii) plasmodia of Badhamia utricularis [14]. They try to occupy free attractants antagonistically. So, if an attractant is occupied by the plasmodium of Physarum polycephalum, it cannot be occupied by the plasmodium of Badhamia utricularis and if it is occupied by the plasmodium of Badhamia utricularis, it cannot be occupied by the plasmodium of Physarum polycephalum. In this way we observe a competition between two plasmodia.

In order to implement the performative syllogistic in the Go games with Physarum polycephalum and Badhamia utricularis plasmodia, we will interpret data points denoted by appropriate syllogistic letters as black stones (attractants) if we assume that appropriate cells are occupied by the plasmodium of Physarum polycephalum and we will interpret data points denoted by appropriate syllogistic letters as white stones (attractants) if we assume that appropriate cells are occupied by the plasmodium of Badhamia utricularis. A data point $S$ is considered empty for the Black player if and only if an appropriate attractant denoted by $S$ is occupied by the white stone (plasmodium of Badhamia utricularis). A data point $S$ is considered empty for the White player if and only if an appropriate attractant denoted by $S$ is occupied by the black stone (plasmodium of Physarum polycephalum). Let us define syllogistic strings of the form $S P$ with the following interpretation: (i) ' $S$ is $P$ ': $S P$ is true for the Black player if and only if $S$ and $P$ are reachable for each other by the plasmodium of Physarum polycephalum and both $S$ and $P$ are not empty for the Black player, otherwise $S P$ is false; (ii) ' $S$ is $P$ ': $S P$ is true for the White player if and only if $S$ and $P$ are reachable for each other by the plasmodium of Badhamia utricularis and both $S$ and $P$ are not empty for the White player, otherwise $S P$ is false. In other words, $S P$ is true for the Black player (respectively, for the White player) if and only if $S$ and $P$ are not empty for the Black player (respectively, for the White player) and there is a line of non-empty cells for the Black player (respectively, for the White player) between points $S$ and $P$, otherwise $S P$ is false. Using this definition of syllogistic strings, we can define atomic syllogistic propositions as follows:

In the formal performative syllogistic: there exists $A$ such that $A$ is $S$ and for any $A, A S$ is true and $A P$ is true. In the Go game model: there is a black (white) stone in $A$ connected by black (white) stones to $S$ and connected by black (white) stones to $P$. In the Physarum model: a plasmodium of Physarum polycephalum (a plasmodium of Badhamia utricularis) in $A$ occupies $S$ and for any plasmodia $A$ of Physarum polycephalum (for any plasmodia $A$ of Badhamia utricularis) which is a neighbor for $S$ and $P$, there are strings $A S$ and $A P$. This means that we have a massive-parallel occupation of the region by plasmodia of Physarum polycephalum (plasmodia of Badhamia utricularis) where the cells $S$ and $P$ are located.

In the formal performative syllogistic: for any $A$, both $A S$ is false and $A P$ is false. In the Go game model: for any cell $A$ there are no lines of black (white) stones connecting $A$ to $S$ and $A$ to $P$. In the Physarum model: for any plasmodium of Physarum polycephalum (of Badhamia utricularis) $A$ which is a neighbor for $S$ and $P$, there are no strings $A S$ and $A P$. This means that the plasmodium of Physarum polycephalum (of Badhamia utricularis) cannot reach $S$ from $P$ or $P$ from $S$ immediately.

In the formal performative syllogistic: there exists $A$ such that if $A S$ is false, then $A P$ is true. In the Go game model: there exists a cell $A$ with the black (white) stone which is a neighbor for cells $S$ and $P$ such that there is a string $A S$ or there is a string $A P$. In the Physarum model: there exists the plasmodium of Physarum polycephalum (of Badhamia utricularis) $A$ which is a neighbor for $S$ and $P$ such that there is a string $A S$ or there is a string $A P$. This means that the plasmodium of Physarum polycephalum (of Badhamia utricularis) occupies $S$ or $P$, but not the whole region where the cells $S$ and $P$ are located.

In the formal performative syllogistic: for any $A, A S$ is false or there exists $A$ such that $A S$ is false or $A P$ is false. In the Go game model: for any cell $A$ with the black (white) stone which is a neighbor for $S$ and $P$ there is no string $A S$ or there exists a black (white) stone in $A$ which is a neighbor for $S$ and $P$ such that there is no string $A S$ or there is no string AP. In the Physarum model: for any plasmodium of Physarum polycephalum (of Badhamia utricularis) A which is a neighbor for $S$ and $P$ there is no string $A S$ or there exists $A$ which is a neighbor for $S$ and $P$ such that there is no string $A S$ or there is no string $A P$. This means that the plasmodium 
of Physarum polycephalum (of Badhamia utricularis) does not occupy $S$ or there is a neighboring cell which is not connected to $S$ or $P$ by a protoplasmic tube.

Notice that there are the following semantic correlations between propositions in the sense of the Black player and propositions in the sense of the White player:

- $S a P$ is true for the Black player iff $S i P$ is true for the White player with the same cells $S$ and $P$;

- $S o P$ is true for the Black player iff $S e P$ is true for the White player with the same cells $S$ and $P$;

- $S a P$ is false for the Black player iff $S i P$ is false for the White player with the same cells $S$ and $P$;

- $S o P$ is false for the Black player iff $S e P$ is false for the White player with the same cells $S$ and $P$.

Composite propositions are defined in the standard way.

In the performative syllogistic we have the following axioms:

$$
\begin{gathered}
S a P:=(\exists A(A \mathrm{i} s S) \wedge(\forall A(A \mathrm{i} s S \wedge A \mathrm{i} s P))) ; \\
S i P:=\forall A(\neg(A \mathrm{i} s S) \wedge \neg(A \mathrm{i} s P)) ;
\end{gathered}
$$

$$
\begin{aligned}
& S o P:=\neg(\exists A(A \mathrm{i} s S) \vee(\forall A(A \mathrm{i} s P \wedge \text { Ais } S))), \text { i.e. } \\
& (\forall A \neg(A \mathrm{i} s S) \wedge \exists A(\neg(A \mathrm{i} s P) \vee \neg(A \mathrm{i} s S))) ;
\end{aligned}
$$

$$
\begin{aligned}
& S e P:=\neg \forall A(\neg(A \mathrm{i} s S) \wedge \neg(A \mathrm{i} s P)), \text { i.e. } \\
& \exists A(A \mathrm{i} s S \vee A \mathrm{i} s P) .
\end{aligned}
$$

$$
S a P \Rightarrow S e P
$$$$
S a P \Rightarrow P a S
$$

$$
S i P \Rightarrow P i S
$$

$$
S a M \Rightarrow S e P
$$

$$
\mathrm{MaP} \Rightarrow \mathrm{SeP}
$$

$$
(M a P \wedge S a M) \Rightarrow S a P
$$

$$
(M i P \wedge S i M) \Rightarrow S i P \text {. }
$$

The formal properties of this axiomatic system are considered in [9], [10]. In the performative syllogistic we can analyze the collective dimension of behavior. Within this system we can study how the plasmodium of Physarum polycephalum and the plasmodium of Badhamia utricularis occupy all possible attractants in any direction if they can see them. So, this system shows logical properties of a massive-parallel behavior (i.e. the collective dimension of behavior). One of the most significant notions involved in this implementation of the performative syllogistic in plasmodia topology is a neighborhood. We can define a distance for the neighborhood differently, i.e. we can make it broader or narrower. So, from different neighborhoods it will follow that we deal with different 'universes of discourse'.

\section{A. Antagonistic Go Game for Verifying Performative Syllogistic Reasoning}

In the Go game diagrams for the performative syllogistic, the 'universe of discourse' cover cells $x, y$, non- $x$ (which be denoted by $x^{\prime}$ ), non- $y$ (which be denoted by $y^{\prime}$ ):

\begin{tabular}{|l|l|}
\hline$x$ & $y^{\prime}$ \\
\hline$y$ & $x^{\prime}$ \\
\hline
\end{tabular}

where $x, y, x^{\prime}, y^{\prime}$ are neighbor cells containing black stones (interpreted as attractants for Physarum polycephalum) and white stones (interpreted as attractants for Badhamia utricularis), $x^{\prime}$ are all neighbors for $y$ which differ from $x$, and $y^{\prime}$ are all neighbors for $x$ which differ from $y$. Let us consider the Go game from the point of view just of the Black player. Suppose that we have black, white, and grey stones and (i) if a black stone is placed within a cell, this means that "this cell is occupied by the plasmodium of Physarum polycephalum" (i.e. "there is at least one thing in it for the Black player"), (ii) if a white stone is placed within a cell, this means that "this cell is not occupied the plasmodium of Physarum polycephalum" (i.e. "there is not thing in it for the Black player"), (ii) if a grey stone is placed within a cell, this means that "it is not known if this cell is occupied by the plasmodium of Physarum polycephalum" or "it is not known what color of stone placed within a cell is from the point of view of the Black player". All possible combinations of Go game diagrams for atomic propositions within our universe of discourse are pictured in Fig. 10.

The universe of discourse for simulating performative syllogisms by means of Physarum behaviors covers cells $x$, $y, m, x^{\prime}, y^{\prime}, m^{\prime}$ in the following manner:

\begin{tabular}{|l|l|l|l|}
\hline$y^{\prime}$ & $m$ & $m^{\prime}$ & $x^{\prime}$ \\
\hline$m^{\prime}$ & $x$ & $y^{\prime}$ & $m$ \\
\hline$m$ & $y$ & $x^{\prime}$ & $m^{\prime}$ \\
\hline$x$ & $m^{\prime}$ & $m$ & $y$ \\
\hline
\end{tabular}

The motion of plasmodium starts from one of the central cells $\left(x, y, x^{\prime}, y^{\prime}\right)$ and goes towards one of the four directions (northwest, southwest, northeast, southeast). The Go game diagram for syllogistic conclusions is as follows:

\begin{tabular}{|l|l|}
\hline$x$ & $m^{\prime}$ \\
\hline$m$ & $x^{\prime}$ \\
\hline
\end{tabular}

Some examples of performative syllogistic conclusions are regarded in Fig. 11.

Thus, the performative syllogistic allows us to study different zones containing attractants for Physarum 
polycephalum and Badhamia utricularis if they are connected by protoplasmic tubes homogenously.

A model $\mathrm{M}^{\prime}=\left\langle M^{\prime},|\cdot|_{x}\right\rangle$ for the performative syllogistic, where $M^{\prime}$ is the set of attractants and $\mid X \mathrm{I}_{x} \subseteq M^{\prime}$ is a meaning of syllogistic letter $X$ which is understood as all attractants reachable for the plasmodium of Physarum polycephalum from the point $x$, is defined as follows:

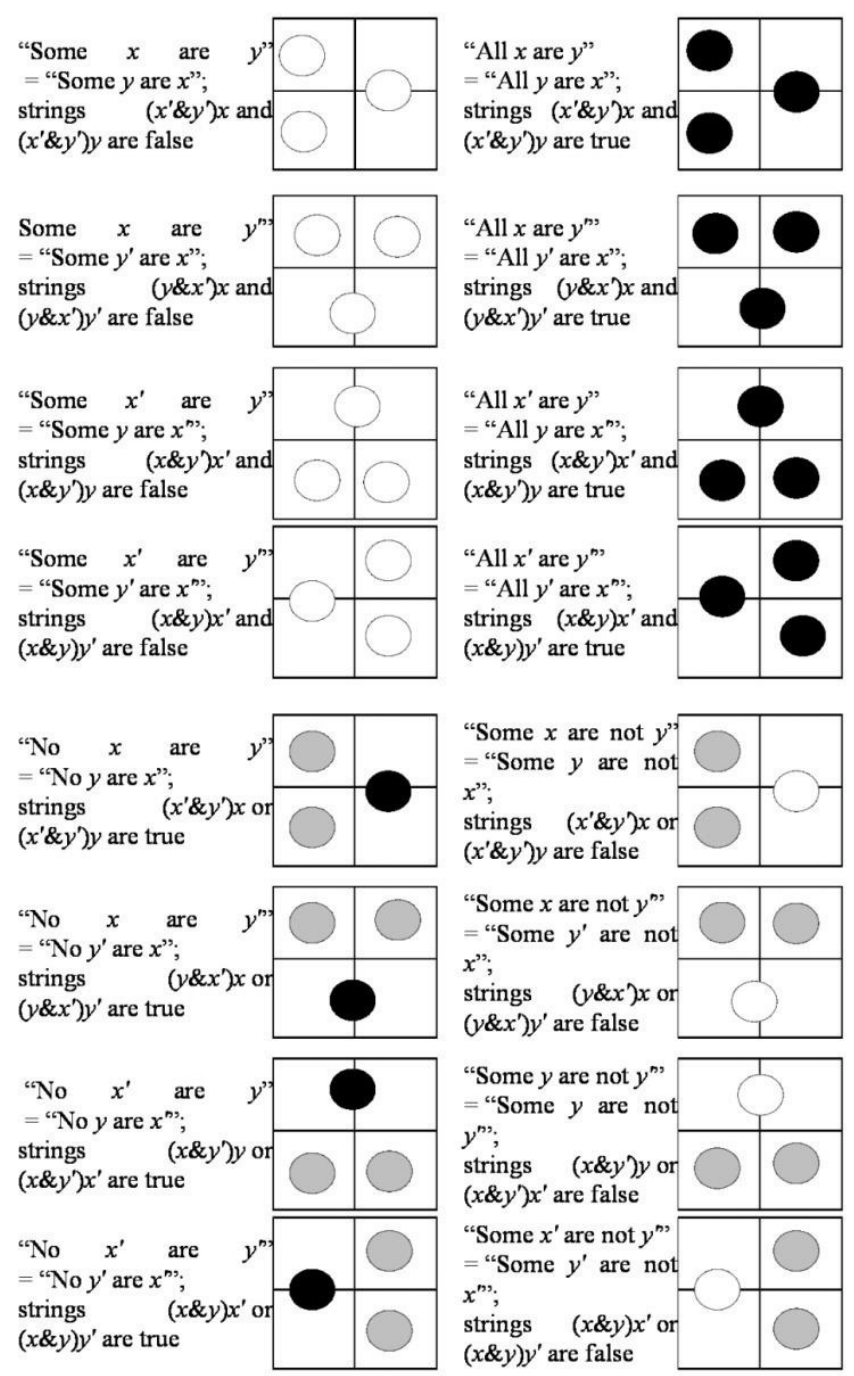

Fig. 10 The Go game diagrams for premises of performative syllogisms. Strings of the form $\left(x^{\prime} \& y^{\prime}\right) x$ mean that in cells $x^{\prime}$ and $y^{\prime}$ there are neighbors $A$ for $x$ such that $A x$, i.e. $\left(x^{\prime} \& y^{\prime}\right)$ is a metavariable in $\left(x^{\prime} \& y^{\prime}\right) x$ that is used to denote all attractants of $x^{\prime}$ and $y^{\prime}$ which are neighbors for the attractant of $x$

$$
\mathrm{M}^{\prime} \mid=\text { All } x \text { are } y \text { iff }|X|_{x} \neq \varnothing,|X|_{y} \neq \varnothing \text {, and }|X|_{x} \cap
$$

$|X|_{y} \neq \varnothing$, more precisely both $\left(x^{\prime} \& y^{\prime}\right) x$ and $\left(x^{\prime} \& y^{\prime}\right) y$ hold in $\mathrm{M}^{\prime}$, i.e. the plasmodium of Physarum polycephalum can move from neighbors of $y$ to $x$ and it can move from neighbors of $x$ to $y$ (we can place black stones in the line from neighbors of $y$ to $x$ and from neighbors of $x$ to $y$ );
$\mathrm{M}^{\prime} \mid=$ Some $x$ are $y$ iff $y \notin|X|_{x}$ and $x \notin|X|_{y}$, more precisely neither $\left(x^{\prime} \& y^{\prime}\right) x$ nor $\left(x^{\prime} \& y^{\prime}\right) y$ hold in $\mathrm{M}^{\prime}$, i.e. the plasmodium of Physarum polycephalum cannot move from neighbors of $y$ to $x$ and it cannot move from neighbors of $x$ to $y$ (we can place white stones in the line from neighbors of $y$ to $x$ and from neighbors of $x$ to $y$ );

All $x$ are All $y$ are $m$.

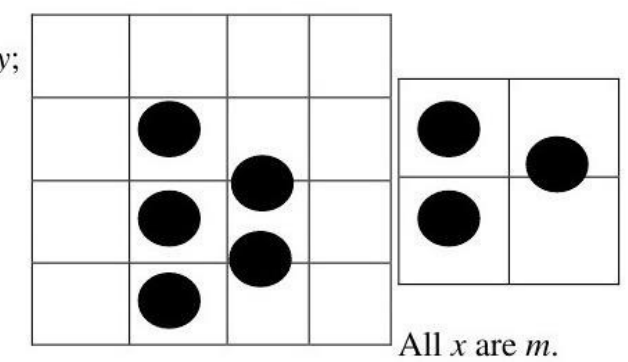

All $x^{\prime}$ are $y$ All $y$ are $m^{\prime}$.
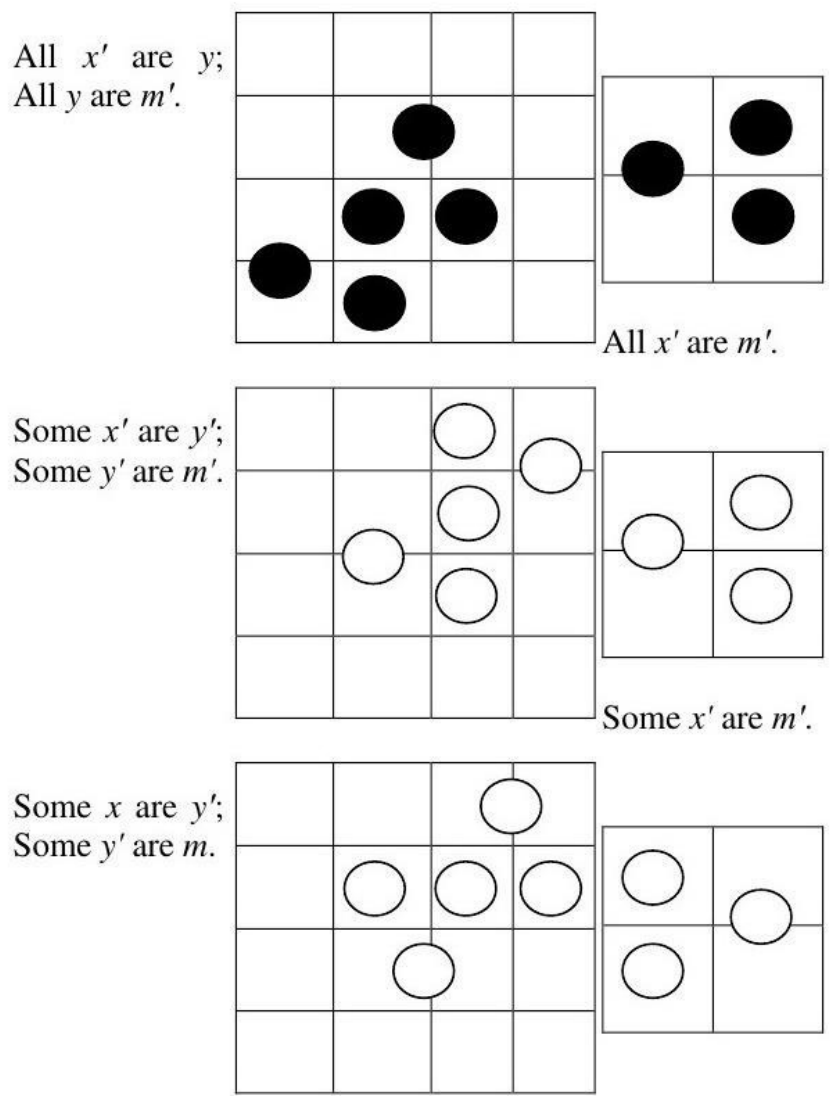

Some $x$ are $m$.

Fig. 11 The Go game diagrams for performative syllogisms with true conclusions from the point of view of the Black player

$\mathrm{M}^{\prime} \mid=$ No $x$ are $y$ iff $y \in|X|_{x}$ or $x \in|X|_{y}$, more precisely $\left(x^{\prime} \& y^{\prime}\right) x$ or $\left(x^{\prime} \& y^{\prime}\right) y$ hold in $\mathrm{M}^{\prime}$, i.e. the plasmodium of Physarum polycephalum can move from neighbors of $y$ to $x$ or it can move from neighbors of $x$ to $y$ (we can place black stones in the line from neighbors of $y$ to $x$ or from neighbors of $x$ to $y$ ); 
$\mathrm{M}^{\prime} \mid=$ Some $x$ are not $y$ iff $y \notin|X|_{x}$ or $x \notin|X|_{y}$, more precisely $\left(x^{\prime} \& y^{\prime}\right) x$ or $\left(x^{\prime} \& y^{\prime}\right)$ do not hold in $\mathrm{M}^{\prime}$, i.e. the plasmodium of Physarum polycephalum cannot move from neighbors of $y$ to $x$ or it cannot move from neighbors of $x$ to $y$ (we can place white stones in the line from neighbors of $y$ to $x$ or from neighbors of $x$ to $y$ );

$$
\begin{aligned}
& \mathrm{M}^{\prime} \mid=p \wedge q \text { iff } \mathrm{M}^{\prime} \mid=p \text { and } \mathrm{M}^{\prime} \mid=q ; \\
& \mathrm{M}^{\prime} \mid=p \vee q \text { iff } \mathrm{M}^{\prime} \mid=p \text { or } \mathrm{M}^{\prime} \mid=q ; \\
& \mathrm{M}^{\prime} \mid=\neg p \text { iff it is false that } \mathrm{M}^{\prime} \mid=p
\end{aligned}
$$

Proposition 3: The performative syllogistic is sound and complete in $\mathrm{M}^{\prime}$.

For more details on formal properties of performative syllogistic, please see [9], [10]. This syllogistic describes the logic of plasmodium propagation in all possible directions. For the implementation of this syllogistic we do not need repellents. It is a natural system. The performative syllogistic as a Go game is an antagonistic game, where two players draw own conclusions without any coalition (see Fig. 10).

\section{B. Examples of Performative-Syllogistic Go Game}

Let us also examine a game of Go at time step 10 to provide an example for performative syllogistic. Let this game be shown in Figure 12. As usual, each Voronoi cell is denoted from $S_{1,1}$ to $S_{18,18}$. In the universe of Fig. 12 there are no universal affirmative propositions and particular affirmative propositions. We face only universal negative propositions and particular negative propositions such as 'No $S_{4,9}^{\prime}$ are $S_{4,10}^{\prime}$ ', where $S_{4,9}^{\prime}$ are neighbors for $S_{4,10}$ differing from $S_{4,9}$ and $S_{4,10}^{\prime}$ are neighbors for $S_{4,9}$ differing from $S_{4,10}$, and 'Some $S_{4,11}^{\prime}$ are not $S_{5,11}^{\prime}$ ', where $S_{4,11}^{\prime}$ are neighbors for $S_{5,11}$ differing from $S_{4,11}$ and $S_{5,11}^{\prime}$ are neighbors for $S_{4,11}$ differing from $S_{5,11}$.

In the universe of Figure 13 there is a universal affirmative proposition: 'All $S_{6,5}$ are $S_{6,6}$ ', and a particular affirmative proposition: 'Some $S_{5,8}$ are $S_{5,9}$ '.

\section{V.CONCLUSION}

We have just shown that we can simulate the plasmodium motion as a Go game, where black stones are interpreted as attractants and white stones as repellents. We can consider configurations of stones as spatial reasoning. If we implement the Aristotelian syllogistic, we need a coalition of two players. If we implement the performative syllogistic [9], [10], we deal with an antagonistic game. In the latter case black stones are interpreted as attractants for Physarum polycephalum and white stones as attractants for Badhamia utricularis.

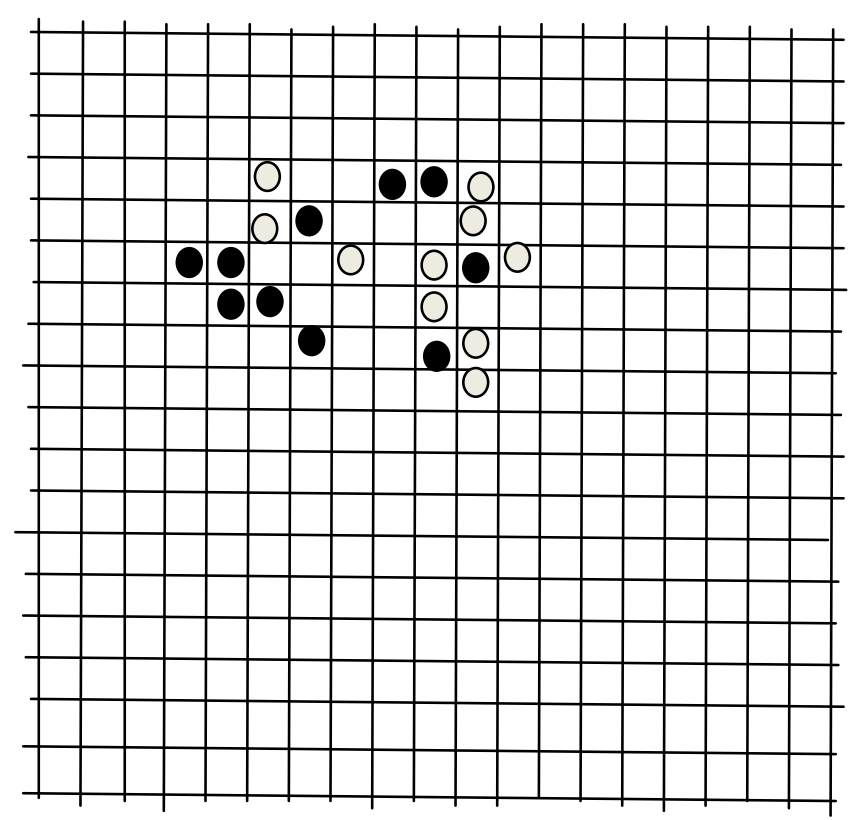

Fig. 12 The performative syllogistic Go game 1 at time step 10. The black stones are attractants occupied by the plasmodium of Physarum polycephalum and the white stones are attractants occupied by the plasmodium of Badhamia utricularis. So, we construct syllogisms from the point of view of the Black player

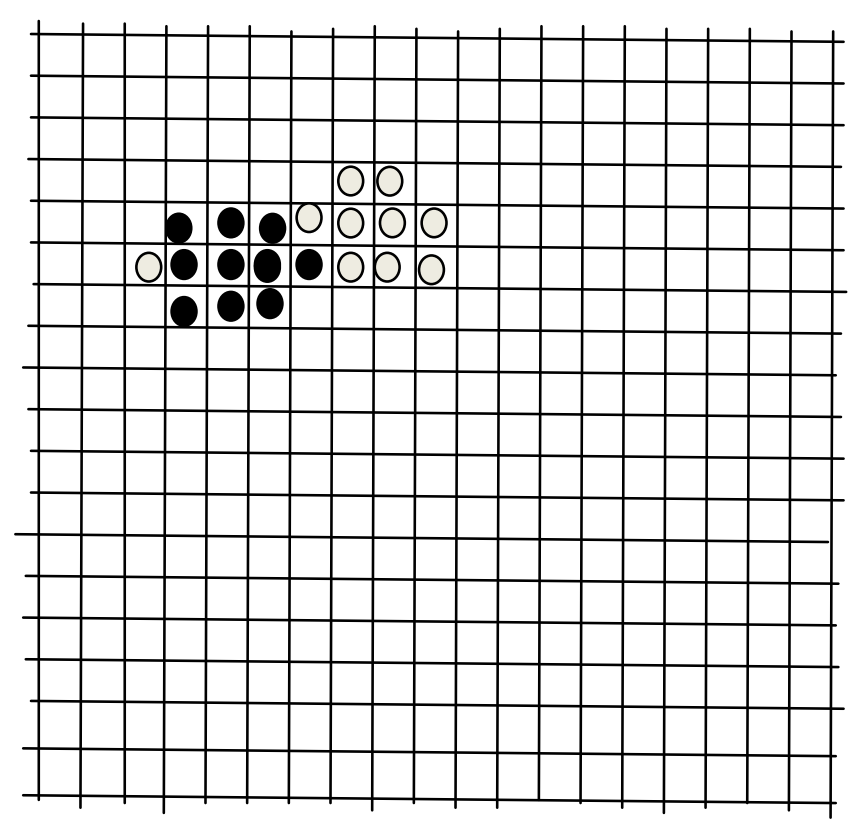

Fig. 13 The performative syllogistic Go game 2 at time step 10. The black stones are attractants occupied by the plasmodium of Physarum polycephalum and the white stones are attractants occupied by the plasmodium of Badhamia utricularis. We build syllogisms from the point of view of the Black player

\section{REFERENCES}

[1] A. Adamatzky, V. Erokhin, M. Grube, Th. Schubert, A. Schumann, "Physarum Chip Project: Growing Computers From Slime Mould," International Journal of Unconventional Computing, vol. 8, no. 4, pp. 319-323, 2012. 
[2] A. Adamatzky, Physarum Machines: Computers from Slime Mould (World Scientific Series on Nonlinear Science, Series A). World Scientific Publishing Company, 2010.

[3] J. Łukasiewicz, Aristotle's Syllogistic From the Standpoint of Modern Formal Logic. Oxford Clarendon Press, 2nd edition, 1957.

[4] T. Nakagaki, H. Yamada, A. Toth, Maze-solving by an amoeboid organism. Nature, vol. 407, pp. 470-470, 2000.

[5] T. Nakagaki, H. Yamada, and A. Tothm, "Path finding by tube morphogenesis in an amoeboid organism," Biophysical Chemistry, vol. 92 , pp. $47-52,2001$

[6] T. Nakagaki, M. Iima, T. Ueda, Y. Nishiura, T. Saigusa, A. Tero, R. Kobayashi, K. Showalter, "Minimum-risk path finding by an adaptive amoeba network," Physical Review Letters, vol. 99, pp. 68104, 2007.

[7] T. Saigusa, A. Tero, T. Nakagaki, Y. Kuramoto, "Amoebae Anticipate Periodic Events," Phys. Rev. Lett, vol. 100, no. 1, 2008.

[8] A. Schumann, L. Akimova, "Simulating of Schistosomatidae (Trematoda: Digenea) Behavior by Physarum Spatial Logic," Annals of Computer Science and Information Systems, Volume 1. Proceedings of the 2013 Federated Conference on Computer Science and Information Systems. IEEE Xplore, 2013, pp. 225-230.

[9] A. Schumann, "On Two Squares of Opposition: the Lesniewski's Style Formalization of Synthetic Propositions," Acta Analytica, vol. 28, pp. 71-93, 2013.

[10] A. Schumann, "Two Squares of Opposition: for Analytic and Synthetic Propositions," Bulletin of the Section of Logic, vol. 40, no. 3/4, pp. 165-178, 2011.

[11] A. Schumann, "Payoff Cellular Automata and Reflexive Games," Journal of Cellular Automata, vol. 9, no. 4, pp. 287-313, 2014.
[12] A. Schumann, A. Adamatzky, "Physarum Spatial Logic," New Mathematics and Natural Computation, vol. 7, no. 3, pp. 483-498, 2011.

[13] A. Schumann, K. Pancerz, "Towards an Object-Oriented Programming Language for Physarum Polycephalum Computing," in Proceedings of the Workshop on Concurrency, Specification and Programming (CS\&P'2013), Warsaw, Poland, September 25-27, pp. $389-397,2013$

[14] A. Schumann, K. Pancerz, A. Adamatzky, and M. Grube, "BioInspired Game Theory: The Case of Physarum Polycephalum," in $8^{\text {th }}$ International Conference on Bio-inspired Information and Communications Technologies (formerly BIONETICS). ICST, 2015, doi: http://dx.doi.org/10.4108/icst.bict.2014.257869

[15] T. Shirakawa, Y.-P. Gunji, and Y. Miyake, "An associative learning experiment using the plasmodium of Physarum polycephalum," Nano Communication Networks, vol. 2, pp. 99-105, 2011.

[16] R. Smith, "Completeness of an ecthetic syllogistic," Notre Dame J. Formal Logic, vol. 24, no. 2, pp. 224-232, 1983.

[17] A. Tero, T. Nakagaki, K. Toyabe, K. Yumiki, R. Kobayashi, "A Method Inspired by Physarum for Solving the Steiner Problem," IJUC, vol. 6, no. 2, pp. 109-123, 2010.

[18] S. Tsuda, M. Aono, and Y.P. Gunji, "Robust and emergent Physarumcomputing," BioSystems, vol. 73, pp. 45-55, 2004.

[19] Sh. Watanabe, A. Tero, A. Takamatsu, T. Nakagaki, "Traffic optimization in railroad networks using an algorithm mimicking an amoeba-like organism, Physarum plasmodium," Biosystems, vol. 105, no. 3, pp. 225-232, 2011. 